\title{
A mixture theory for geophysical fluids
}

\author{
A. C. Eringen \\ 15 Red Tail Drive, Littleton, Co., 80126-5001, USA \\ Received: 21 January 2003 - Accepted: 13 March 2003 - Published: 25 February 2004 \\ Part of Special Issue "Dedicated to Prof. A. D. Kirwan Jr. on the occasion of his 70th birthday"
}

\begin{abstract}
A continuum theory is developed for a geophysical fluid consisting of two species. Balance laws are given for the individual components of the mixture, modeled as micropolar viscous fluids. The continua allow independent rotational degrees of freedom, so that the fluids can exhibit couple stresses and a non-symmetric stress tensor. The second law of thermodynamics is used to develop constitutive equations. Linear constitutive equations are constituted for a heat conducting mixture, each species possessing separate viscosities. Field equations are obtained and boundary and initial conditions are stated. This theory is relevant to an atmospheric mixture consisting of any two species from rain, snow and/or sand. Also, this is a continuum theory for oceanic mixtures, such as water and silt, or water and oil spills, etc.
\end{abstract}

\section{Introduction}

In the atmosphere, many complex physical phenomena can occur. For example, simultaneously, it may be raining and snowing. Tornadoes or hurricanes may lift sands, mixing with rain or snow. In these cases, we have a mixture of two different fluids. We consider snow, sand and rain as fluids. Although ice and sand grains are solids by themselves, we must treat each species as a liquid, since there is no cohesion among the grains as in a solid. The friction between rain, snow or sand, falling through the saturated air, attributes viscosities to the species. We do not consider the air as a third species, but rather as an agent that allows the fluid assumption for the species to be plausible. Alternatively, air, and either rain, snow or sand, may be considered to constitute a binary mixture of two fluids.

In the case of oceanography, the mixture of water and silt may compose the two species of the mixture. When oil spills

Correspondence to: A. C. Eringen

(103265.462@compuserve.com) occur in a region of ocean, oil and water may be considered as the members of the mixture, in that region.

Most studies in geophysical fluid dynamics are based on the Navier-Stokes (N-S) equations, with the inclusion of the Coriolis acceleration. The Navier-Stokes equations assume the atmosphere as a single fluid. Moreover, in the N-S equations, the effects of couple stresses and intrinsic rotations are ignored. These effects may become important in some instances, e.g. tornadoes, hurricanes, tumbling ice or sand.

Kirwan and Chang (1976) have demonstrated the influence of couple stress in the Ekman problem, in the case of a single micropolar fluid.

Literature is extensive on the mixture theories. Some of the early references are quoted by Bowen (1976), up to 1976, and more recent ones by Eringen (1994). However, these are concerned with nonpolar materials. Twiss and Eringen $(1971,1972)$ gave a theory of mixtures for micromorphic materials and investigated propagation of waves in two-constituent micropolar elastic solids. Several new elastic wave branches predicted by this theory are in accordance with the phonon dispersion experiments. These branches are not predicted by the mixture models based on the classical elastic solids. Consequently, the present theory, too, is expected to exhibit some new physical phenomena that are not in the domain of a mixture theory based on the N-S equations.

1. The raison d'être of the present paper stems from these observations.

In Sect. 2, we discuss briefly, the kinematics of a mixture. Balance laws are given in Sect. 3. Section 4 is concerned with the development of the constitutive equations and the thermodynamic restrictions required on the viscosity moduli. In Sect. 5, we obtain the field equations and state the boundary and initial conditions. Here, we also give the restrictions when fluids are incompressible. In Sect. 6, as a special case, we derive the field equations of a binary mixture of classical fluids. 


\section{Kinematics}

We consider a binary mixture of two continuous fluent bodies $\mathrm{B}^{\alpha}, \alpha=1,2$. In the case of atmospheric activities, $\mathrm{B}^{1}$ may represent the rain, and $\mathrm{B}^{2}$ the snow or sand. In an ocean atmosphere, they may represent water and silt. We consider that, continuous bodies $\mathrm{B}^{\alpha}$, occupy a region of the threedimensional space. A material point, in the $\alpha^{\text {th }}$ species, at the natural state, is denoted by $\boldsymbol{X}^{\alpha}$, in Cartesian coordinates. The motion of $\boldsymbol{X}^{\alpha}$, at time $t$, is represented by a mapping of $\boldsymbol{X}^{\alpha}$, to a spatial point $\boldsymbol{x}$ :

$\boldsymbol{x}=\chi^{\alpha}\left(\boldsymbol{X}^{\alpha}, t\right)$,

which is assumed to possess the unique inverse

$\boldsymbol{X}^{\alpha}=\chi^{-1 \alpha}(\boldsymbol{x}, t)$.

The velocity and the acceleration of $\boldsymbol{X}^{\alpha}$, at time $t$, are given by

$$
\begin{gathered}
\boldsymbol{v}^{\alpha}=\boldsymbol{x}^{\prime \alpha}=\frac{\partial \chi^{\alpha}\left(X^{\alpha}, t\right)}{\partial t}, \\
\boldsymbol{a}^{\alpha}=\boldsymbol{x}^{\prime \prime \alpha}=\frac{\partial^{2} \chi^{\alpha}\left(X^{\alpha}, t\right)}{\partial t^{2}} .
\end{gathered}
$$

As usual, the material time derivative of a function $\psi(x, t)$ is denoted by

$\dot{\psi}=\left.\frac{\partial \psi}{\partial t}\right|_{\boldsymbol{X}}=\frac{\partial \psi(\boldsymbol{x}, t)}{\partial t}+\psi_{, k} \dot{x}_{k}(\boldsymbol{x}, t)$,

where and henceforth, we employ the summation convention on repeated Latin indices, and an index following a comma for a partial derivative with respect to $x_{k}$, e.g.

$\psi_{, k} \dot{x}_{k}=\frac{\partial \psi}{\partial x_{1}} \dot{x}_{1}+\frac{\partial \psi}{\partial x_{2}} \dot{x}_{2}+\frac{\partial \psi}{\partial x_{3}} \dot{x}_{3}$.

The material derivative of a function $\psi\left[\chi^{\alpha}\left(X^{\alpha}, t\right), t\right]$, following the motion of the constituent $\mathrm{B}^{\alpha}$, is defined by

$\psi^{\prime \alpha}=\left.\frac{\partial \psi\left[\chi^{\alpha}\left(X^{\alpha}, t\right), t\right]}{\partial t}\right|_{X^{\alpha}}=\frac{\partial \psi(\boldsymbol{x}, t)}{\partial t}+\psi_{, k} x_{k}^{\prime \alpha}$.

From Eq. (4) and Eq. (5), it follows that

$\psi^{\prime \alpha}-\dot{\psi}=(\nabla \psi) \cdot\left(\dot{\boldsymbol{x}}^{\prime \alpha}-\dot{\boldsymbol{x}}^{\alpha}\right)$.

The mass density of the constituent $\mathrm{B}^{\alpha}$ is denoted by $\rho^{\alpha}(\boldsymbol{x}, t)$. The density $\rho(\boldsymbol{x}, t)$ and the velocity $\boldsymbol{v}(\boldsymbol{x}, t)$ of the mixture are given by

$\rho(\boldsymbol{x}, t)=\sum_{\alpha} \rho^{\alpha}(\boldsymbol{x}, t), \quad \dot{\boldsymbol{x}}=\boldsymbol{v}=\frac{1}{\rho} \sum_{\alpha} \rho^{\alpha} \boldsymbol{v}^{\alpha}$.

The barycentric velocity is defined by

$\overline{\boldsymbol{v}}^{\alpha}=\boldsymbol{v}^{\alpha}-\boldsymbol{v}$

The mass concentration $c^{\alpha}$ of the constituent $\mathrm{B}^{\alpha}$, at $(\boldsymbol{x}, t)$ is

$c^{\alpha}(\boldsymbol{x}, t)=\rho^{\alpha} / \rho$.
From Eq. 7 and Eq. 9, it follows that

$\sum_{\alpha} c^{\alpha}=1$.

Since rain drops, snow and sand undergo translations and rotations, the mixture continua must possess rotational degrees of freedom. This suggests that we consider each species as a micropolar continuum. In a micropolar continuum, a material point is endowed with a director, so that rotational degrees of freedom arise from the rotation of the director. For micropolar fluids, this is represented by gyration vector $v_{k}$. Micropolar fluid continua possess two deformationrate tensors (c.f. Eringen, 2001):

$a_{k l}=v_{l, k}+\epsilon_{l k m} v_{m}, \quad b_{k l}=v_{k, l}$.

Consequently, each species of the mixture is endowed with two deformation tensors

$a_{k l}^{\alpha}=v_{l, k}^{\alpha}+\epsilon_{l k m} v_{m}^{\alpha}, \quad b_{k l}^{\alpha}=v_{k, l}^{\alpha}$.

\section{Balance laws}

We assume that no chemical reactions take place between the two constituents of the mixture.

The balance laws for each constituent $\mathrm{B}^{\alpha}$, consist of: conservation of mass, balance of momentum, balance of moment of momentum, and conservation of energy.

Conservation of Mass

$\frac{\partial \rho^{\alpha}}{\partial t}+\nabla \cdot\left(\rho^{\alpha} \boldsymbol{v}^{\alpha}\right)=0 \quad$ or $\quad \rho^{\alpha}\left|\operatorname{det} \boldsymbol{F}^{\alpha}\right|=\rho_{0}^{\alpha}$,

where $\rho_{0}^{\alpha}$ is the mass density in the natural state, and $\boldsymbol{F}^{\alpha}$ is the deformation gradient, defined by

$\boldsymbol{F}^{\alpha}=\nabla \chi^{\alpha}\left(X^{\alpha}, t\right) \quad$ or $\quad F_{k K}^{\alpha}=\frac{\partial x_{k}}{\partial X_{K}^{\alpha}}$.

Summing Eq. (13) over $\alpha$, we obtain the equation of conservation of mass for the mixture:

$\frac{\partial \rho}{\partial t}+\nabla \cdot(\rho \boldsymbol{v})=0$.

Another useful form of Eq. (13) follows upon using Eq. (7) and Eq. (8):

$\rho \dot{c}^{\alpha}+\nabla \cdot\left(\rho^{\alpha} \overline{\boldsymbol{v}}^{\alpha}\right)=0$.

Balance of Momentum

$\rho^{\alpha} \boldsymbol{x}^{\prime \prime \alpha}=\nabla \cdot \boldsymbol{t}^{\alpha}+\rho^{\alpha} \boldsymbol{f}^{\alpha}+\hat{\boldsymbol{p}}^{\alpha}$,

$\sum_{\alpha} \hat{\boldsymbol{p}}^{\alpha}=\mathbf{0}$,

where, $t_{k l}^{\alpha}, f_{k}^{\alpha}$ and $\hat{p}_{k}^{\alpha}$ are, respectively, the stress tensor, the body force density, and the force exerted on the $\alpha$ th constituent from the other constituent.

Balance of Moment of Momentum

$\rho^{\alpha} j^{\alpha} v_{l}^{\prime \alpha}=m_{k l, k}^{\alpha}+\epsilon_{l m n} t_{m n}^{\alpha}+\rho^{\alpha} l_{l}^{\alpha}+\hat{m}_{l}^{\alpha}$, 
$\sum_{\alpha} \hat{m}_{l}^{\alpha}=0$,

where, $\rho^{\alpha} j^{\alpha}, v_{l}^{\alpha}, m_{k l}^{\alpha}, \rho^{\alpha} l_{l}^{\alpha}$ and $\hat{m}_{l}$ are, respectively, microinertia density, microrotation-rate (gyration), body couple density, and the couple exerted on $\alpha$ th constituent from the other constituent. $\epsilon_{l m n}$ is the alternating tensor. Note that, when $j^{\alpha}, m_{k l}^{\alpha}$ and $l_{l}^{\alpha}$ vanish, Eq. (19) reduces to the classical form

$\epsilon_{l m n} t_{m n}^{\alpha}+\hat{m}_{l}^{\alpha}=0$.

If we sum this over $\alpha$, we arrive at the symmetry of the mixture stress tensor $t_{k l}=t_{l k}$. Thus, Eq. (19) expresses the balance of spin inertia.

Conservation of Energy

$\rho^{\alpha} \hat{\epsilon}^{\alpha}=t_{k l}^{\alpha} a_{k l}^{\alpha}+m_{k l}^{\alpha} b_{l k}^{\alpha}+q_{k, k}^{\alpha}+\rho^{\alpha} h^{\alpha}+\hat{\epsilon}^{\alpha}$,

Subject to

$\sum_{\alpha}\left(\hat{\epsilon}^{\alpha}+\hat{\boldsymbol{p}}^{\alpha} \cdot \overline{\boldsymbol{v}}^{\alpha}+\hat{\boldsymbol{m}}^{\alpha} \cdot \overline{\boldsymbol{v}}^{\alpha}\right)=0$,

where

$\overline{\boldsymbol{v}}^{\alpha}=\boldsymbol{v}^{\alpha}-\boldsymbol{v}, \quad \overline{\boldsymbol{v}}^{\alpha}=\boldsymbol{v}^{\alpha}-\boldsymbol{v}$.

Here, $\epsilon^{\alpha}, \boldsymbol{q}^{\alpha}$ and $h^{\alpha}$ are, respectively, the internal energy density, the heat vector, and the energy source of $\alpha$ th species. $\hat{\epsilon}^{\alpha}$ denotes the transfer of energy to $\alpha$ th species from the other species.For the derivation of these equations, we refer the reader to Twist and Eringen (1994), Eringen (2001), Eringen and Ingram (1965) and Bowen (1976).

We consider that both species have the same temperature. Certainly this is not true for the rain and ice mixture, except perhaps at the melting point. But, for a mixture consisting of rain and sand, it can be valid. In this case, we need only a single energy equation, namely that of the mixture. To this end, we sum Eq. (22) over $\alpha$, use Eq. (23) and

$\boldsymbol{q}=\sum_{\alpha}\left(\boldsymbol{q}^{\alpha}-\rho^{\alpha} \epsilon^{\alpha} \overline{\boldsymbol{v}}^{\alpha}\right), \quad \rho h=\sum_{\alpha} \rho^{\alpha} h^{\alpha}$.

Using the expression

$$
\begin{aligned}
& \sum_{\alpha} \rho^{\alpha} \dot{\psi}^{\alpha}=\rho \dot{\psi}+\sum_{\alpha} \nabla \cdot\left(\rho^{\alpha} \psi^{\alpha} \overline{\boldsymbol{v}}^{\alpha}\right), \\
& \rho \psi=\sum_{\alpha} \rho^{\alpha} \psi^{\alpha},
\end{aligned}
$$

we obtain

$$
\begin{aligned}
\rho \dot{\epsilon}=\nabla & \cdot \boldsymbol{q}+\rho h \\
& +\sum_{\alpha}\left(t_{k l}^{\alpha} a_{k l}^{\alpha}+m_{k l}^{\alpha} b_{l k}^{\alpha}-\hat{\boldsymbol{p}}^{\alpha} \cdot \overline{\boldsymbol{v}}^{\alpha}-\hat{\boldsymbol{m}}^{\alpha} \cdot \overline{\boldsymbol{v}}^{\alpha}\right) .
\end{aligned}
$$

Using Eq. (18) and Eq. (20), we have

$\sum_{\alpha=1}^{2} \hat{\boldsymbol{p}}^{\alpha} \cdot \overline{\boldsymbol{v}}^{\alpha}=\hat{\boldsymbol{p}}^{1} \cdot \overline{\boldsymbol{v}}^{12}, \quad \sum_{\alpha=1}^{2} \hat{\boldsymbol{m}}^{\alpha} \cdot \overline{\boldsymbol{v}}^{\alpha}=\hat{\boldsymbol{m}}^{1} \cdot \overline{\boldsymbol{v}}^{12}$,

where

$\overline{\boldsymbol{v}}^{12}=\boldsymbol{v}^{1}-\boldsymbol{v}^{2}, \quad \overline{\boldsymbol{v}}^{12}=\boldsymbol{v}^{1}-\boldsymbol{v}^{2}$.
With this, Eq. (27) becomes

$\begin{aligned} \rho \dot{\epsilon}= & \nabla \cdot \boldsymbol{q}+\rho h \\ & +\sum_{\alpha}\left(t_{k l}^{\alpha} a_{k l}^{\alpha}+m_{k l}^{\alpha} b_{l k}^{\alpha}\right)-\hat{\boldsymbol{p}}^{1} \cdot \overline{\boldsymbol{v}}^{12}-\hat{\boldsymbol{m}}^{1} \cdot \overline{\boldsymbol{v}}^{12} .\end{aligned}$

We introduce Helmholtz's free energy $\psi$ by

$\epsilon=\psi+\theta \eta$,

where, $\theta>0(\inf \theta=0)$ is the absolute temperature, and $\eta$ is the entropy density. With this, the energy balance law Eq. (30) takes the form

$$
\begin{gathered}
\rho \dot{\psi}+\rho \theta \dot{\eta}+\rho \dot{\theta} \eta-\nabla \cdot \boldsymbol{q}-\rho h-\sum_{\alpha}\left(t_{k l}^{\alpha} a_{k l}^{\alpha}+m_{k l}^{\alpha} b_{l k}^{\alpha}\right) \\
+\hat{\boldsymbol{p}}^{1} \cdot \overline{\boldsymbol{v}}^{12}+\hat{\boldsymbol{m}}^{1} \cdot \overline{\boldsymbol{v}}^{12}=0 .
\end{gathered}
$$

To the balance laws, we adjoin the second law of thermodynamics,

$\rho \dot{\eta}-\nabla \cdot\left(\frac{\boldsymbol{q}}{\theta}\right)-\frac{\rho h}{\theta} \geq 0$.

By means of Eq. (32), we eliminate $\rho h / \theta$ from Eq. (33), to obtain

$$
\begin{gathered}
-\rho(\dot{\psi}+\eta \dot{\theta})+\sum_{\alpha}\left(t_{k l}^{\alpha} a_{k l}^{\alpha}+m_{k l}^{\alpha} b_{l k}^{\alpha}\right)-\hat{\boldsymbol{p}}^{1} \cdot \overline{\boldsymbol{v}}^{12} \\
-\hat{\boldsymbol{m}}^{1} \cdot \overline{\boldsymbol{v}}^{12}+\frac{\boldsymbol{q} \cdot \boldsymbol{\nabla} \theta}{\theta} \geq 0 .
\end{gathered}
$$

This is the generalized Clausius-Duhem inequality. It is fundamental to the development of the constitutive equations.

\section{Constitutive equations}

According to the causality hypothesis, Eringen (1980), the independent variables are

$Y=\left(\theta, \rho^{\alpha}, a_{k l}^{\alpha}, b_{k l}^{\alpha}, \overline{\boldsymbol{v}}^{12}, \overline{\boldsymbol{v}}^{12}, \theta_{, k}\right), \quad \alpha=1,2$

where,

$\overline{\boldsymbol{v}}^{12}=\boldsymbol{v}^{1}-\boldsymbol{v}^{2}, \quad \overline{\boldsymbol{v}}^{12}=\boldsymbol{v}^{1}-\boldsymbol{v}^{2}$.

Dependent variables

$Z=\left(\psi, \eta, t_{k l}^{\alpha}, m_{k l}^{\alpha},-\hat{\boldsymbol{p}}^{1},-\hat{\boldsymbol{m}}^{1}, \boldsymbol{q}\right)$

are considered to be functions of $Y$.

We observe that, all independent variables are frameindependent, except for $\overline{\boldsymbol{v}}^{12}$ and $\overline{\boldsymbol{v}}^{12}$. Generally, these latter quantities are not admissible to form a constitutive equation in nonlinear continuum mechanics. However, it appears in classical Darcy's law, and is well accepted in porous media theories. The admissibility of this term, as a linear approximation in continuum mechanics, was noted by Wilmanski (2003). The same argument readily applies to the variable $\overline{\boldsymbol{v}}^{12}$. 
Static variables are $\theta$ and $\rho^{\alpha}$, so that

$\psi=\psi\left(\theta, \rho^{1}, \rho^{2}\right)$.

We calculate

$\dot{\psi}=\frac{\partial \psi}{\partial \theta} \dot{\theta}-\sum_{\alpha} \frac{\partial \psi}{\partial \rho^{\alpha}}\left(\rho^{\alpha} v_{k, k}^{\alpha}\right)$,

where, we used Eq. (13) to replace $\dot{\rho}^{\alpha}$. Substituting Eq. (39) into Eq. (34), we have

$$
\begin{aligned}
-\rho\left(\frac{\partial \psi}{\partial \theta}+\eta\right) \dot{\theta} \\
+\sum_{\alpha}\left[\left(t_{k l}^{\alpha}+\rho \rho^{\alpha} \frac{\partial \psi}{\partial \rho^{\alpha}} \delta_{k l}\right) a_{k l}^{\alpha}+m_{k l}^{\alpha} b_{l k}^{\alpha}\right] \\
-\hat{\boldsymbol{p}}^{1} \cdot \overline{\boldsymbol{v}}^{12}-\hat{\boldsymbol{m}}^{1} \cdot \tilde{\boldsymbol{v}}^{12}+\boldsymbol{q} \cdot \frac{\nabla \theta}{\theta} \geq 0 .
\end{aligned}
$$

This inequality cannot be maintained in one sign, for all independent variations of $\dot{\theta}$ unless

$\eta=-\frac{\partial \psi}{\partial \theta}$

and

$$
\begin{aligned}
\sum_{\alpha}\left({ }_{D} t_{k l}^{\alpha} a_{k l}^{\alpha}+m_{k l}^{\alpha} b_{l k}^{\alpha}\right)-\hat{\boldsymbol{p}}^{1} \cdot \overline{\boldsymbol{v}}^{12}-\hat{\boldsymbol{m}}^{1} \cdot \overline{\boldsymbol{v}}^{12} \\
+\boldsymbol{q} \cdot \frac{\nabla \theta}{\theta} \geq 0
\end{aligned}
$$

where,

$D_{k l}^{\alpha}=t_{k l}^{\alpha}+\pi^{\alpha} \delta_{k l}, \quad \pi^{\alpha}=\rho \rho^{\alpha} \frac{\partial \psi}{\partial \rho^{\alpha}}$.

Here, $\pi^{\alpha}$ is the partial pressure in $\alpha$ th species.

We recognize the thermodynamic forces $\boldsymbol{Y}$ by

$\boldsymbol{Y}=\left(a_{k l}^{\alpha}, b_{l k}^{\alpha}, \bar{v}_{k}^{12}, \bar{v}_{k}^{12}, \theta_{, k} / \theta\right)$,

and thermodynamic fluxes $\boldsymbol{J}$, by

$\boldsymbol{J}=\left({ }_{D} t_{k l}^{\alpha}, m_{k l}^{\alpha},-\hat{p}_{k}^{1},-\hat{m}_{k}^{1}, q_{k}\right)$.

The inequality Eq. (42) may be abbreviated by

$\Gamma=\boldsymbol{Y} \cdot \boldsymbol{J} \geq 0$.
The dissipation (dynamic) part of constitutive equations may be expressed in the form

$\boldsymbol{J}=\mathfrak{J}(\boldsymbol{Y} ; \theta)$.

Edelen (1993) gave a general solution of Eq. (46), in the form

$\mathfrak{J}(\boldsymbol{Y} ; \theta)=\nabla_{\boldsymbol{Y}} \Phi(\boldsymbol{Y} ; \theta)+\boldsymbol{W}(\boldsymbol{Y} ; \theta)$,

where, $\boldsymbol{W}$ is restricted by

$\boldsymbol{W} \cdot \boldsymbol{Y}=0$

and the dissipation potential, $\Phi(\boldsymbol{Y} ; \theta)$ is given by

$\Phi(\boldsymbol{Y} ; \theta)=\int_{0}^{1} \boldsymbol{Y} \cdot \mathfrak{J}(\tau \boldsymbol{Y}, \theta) \frac{d \tau}{\tau}$.

Equation (49) indicates that $\boldsymbol{W}$ do not contribute to the dissipation of energy. In fact, for the linear constitutive equations, $\boldsymbol{W}$ vanish, and we obtain

${ }_{D} \boldsymbol{t}^{\alpha}=\frac{\partial \Phi}{\partial \boldsymbol{a}^{\alpha}}, \quad \boldsymbol{m}^{\alpha}=\frac{\partial \Phi}{\partial \boldsymbol{b}^{\alpha T}}, \quad \hat{\boldsymbol{p}}^{1}=-\hat{\boldsymbol{p}}^{2}=-\frac{\partial \Phi}{\partial \overline{\boldsymbol{v}}^{12}}$,

$\hat{\boldsymbol{m}}^{1}=-\hat{\boldsymbol{m}}^{2}=-\frac{\partial \Phi}{\partial \overline{\boldsymbol{v}}^{12}}, \quad \boldsymbol{q}=\frac{\partial \Phi}{\partial(\nabla \theta / \theta)}$,

where, a superscript $T$ denotes the transpose, e.g. $b_{k l}^{\alpha T}=b_{l k}^{\alpha}$.

The dissipation potential $\Phi$ depends on the invariants of $\boldsymbol{Y}$ and $\theta$. We distinguish pseudo-tensors $\boldsymbol{b}^{\alpha}$ and $\overline{\boldsymbol{v}}^{12}$ from the absolute tensors $\boldsymbol{a}^{\alpha}, \overline{\boldsymbol{v}}^{12}$ and $\nabla \theta / \theta$. For first degree constitutive equations, we need the second degree invariants only. These can be read from a table, Eringen (1980 p. 577):

$\operatorname{tr} \boldsymbol{a}^{\alpha}, \quad \operatorname{tr}\left(\boldsymbol{a}^{\alpha}\right)^{2}, \quad \operatorname{tr}\left(\boldsymbol{a}^{\alpha} \boldsymbol{a}^{\alpha T}\right), \quad \operatorname{tr}\left(\boldsymbol{a}^{1} \boldsymbol{a}^{2 T}\right), \quad\left(\operatorname{tr} \boldsymbol{b}^{\alpha}\right)^{2}$, $\operatorname{tr} \boldsymbol{b}^{\alpha}, \quad \operatorname{tr}\left(\boldsymbol{b}^{\alpha} \boldsymbol{b}^{\alpha T}\right) \operatorname{tr}\left(\boldsymbol{b}^{1} \boldsymbol{b}^{2 T}\right), \quad \overline{\boldsymbol{v}}^{12} \cdot \overline{\boldsymbol{v}}^{12}$,

$\overline{\boldsymbol{v}}^{12} \cdot \overline{\boldsymbol{v}}^{12}, \quad \overline{\boldsymbol{v}}^{12} \cdot \nabla \theta / \theta, \quad K \frac{\nabla \theta \cdot \nabla \theta}{\theta^{2}}, \quad \alpha=1,2$

The dissipation function $\Phi$ may be expressed as

$\Phi=\Phi_{1}+\Phi_{2}$

where,

$$
\begin{aligned}
2 \Phi_{1}= & \sum_{\alpha}\left[\lambda_{\alpha}\left(\operatorname{tr} \boldsymbol{a}^{\alpha}\right)^{2}+\mu_{\alpha} \operatorname{tr}\left(\boldsymbol{a}^{\alpha}\right)^{2}+\left(\mu_{\alpha}+\kappa_{\alpha}\right) \operatorname{tr}\left(\boldsymbol{a}^{\alpha} \boldsymbol{a}^{\alpha T}\right)\right]+2 \sigma \operatorname{tr}\left(\boldsymbol{a}^{1} \boldsymbol{a}^{2 T}\right)+\xi \overline{\boldsymbol{v}}^{12} \cdot \overline{\boldsymbol{v}}^{12}+2 \varsigma \overline{\boldsymbol{v}}^{12} \cdot \frac{\nabla \theta}{\theta} \\
& \quad+K \frac{\nabla \theta \cdot \nabla \theta}{\theta^{2}} \\
& \quad \\
2 \Phi_{2}= & \sum_{\alpha}\left[\alpha_{\alpha}\left(\operatorname{tr} \boldsymbol{b}^{\alpha}\right)^{2}+\beta_{\alpha} \operatorname{tr}\left(\boldsymbol{b}^{\alpha}\right)^{2}+\gamma_{\alpha} \operatorname{tr}\left(\boldsymbol{b}^{\alpha} \boldsymbol{b}^{\alpha T}\right)\right]+2 \tau \operatorname{tr}\left(\boldsymbol{b}^{1} \boldsymbol{b}^{2 T}\right)+\varpi \overline{\boldsymbol{v}}^{12} \cdot \overline{\boldsymbol{v}}^{12}
\end{aligned}
$$

where, $\lambda_{\alpha}, \mu_{\alpha}, \kappa_{\alpha}, \alpha_{\alpha}, \beta_{\alpha}, \gamma_{\alpha}, \xi, \varpi, \varsigma, \sigma, \tau$ and $K$ are material moduli. 
Substituting Eq. (54) into Eq. (51), we obtain the constitutive equations

$$
\begin{aligned}
{ }_{D} \boldsymbol{t}^{\alpha} & =\lambda_{\alpha} \operatorname{tr} \boldsymbol{a}^{\alpha} \mathbf{1}+\mu_{\alpha} \boldsymbol{a}^{\alpha T}+\left(\mu_{\alpha}+\kappa_{\alpha}\right) \boldsymbol{a}^{\alpha}+\sigma\left(\boldsymbol{a}^{1} \delta_{\alpha 2}+\boldsymbol{a}^{2} \delta_{\alpha 1}\right), \\
\boldsymbol{m}^{\alpha} & =\alpha_{\alpha} \operatorname{tr} \boldsymbol{b}^{\alpha} \mathbf{1}+\boldsymbol{\beta}_{\alpha} \boldsymbol{b}^{\alpha}+\gamma_{\alpha} \boldsymbol{b}^{\alpha T}+\tau\left(\boldsymbol{b}^{1 T} \delta_{\alpha 1}+\boldsymbol{b}^{2 T} \delta_{\alpha 1}\right), \quad \alpha=1,2 \\
-\hat{\boldsymbol{p}}^{1} & =\hat{\boldsymbol{p}}^{2}=\xi \overline{\boldsymbol{v}}^{12}+\varsigma \nabla \theta / \theta, \\
-\hat{\boldsymbol{m}}^{1} & =\hat{\boldsymbol{m}}^{2}=\varpi \overline{\boldsymbol{v}}^{12}, \\
\boldsymbol{q} & =\varsigma \overline{\boldsymbol{v}}^{12}+K \nabla \theta / \theta .
\end{aligned}
$$

The dissipation potentials $\Phi_{1}$ and $\Phi_{2}$ must be nonnegative for all independent variations of the independent variable set. For $\boldsymbol{a}^{\alpha}=\mathbf{0}, 2 \Phi_{1}$ reduces to

$\xi \overline{\boldsymbol{v}}^{12} \cdot \overline{\boldsymbol{v}}^{12}+2 \varsigma \overline{\boldsymbol{v}}^{12} \cdot \frac{\nabla \theta}{\theta}+K \frac{\nabla \theta \cdot \nabla \theta}{\theta^{2}} \geq 0$

This will be nonnegative, if and only if

$\xi \geq 0, \quad \xi K-\varsigma^{2} \geq 0$.

From Eq. (54) (with $\boldsymbol{b}^{\alpha}=\mathbf{0}$ ), $\Phi \geq 0$ leads to $\varpi \geq 0$.

For $\overline{\boldsymbol{v}}^{12}=\overline{\boldsymbol{v}}^{12}=\mathbf{0}, \Phi_{1}$ and $\Phi_{2}$ acquire similar quadratic forms. Thus, we need to study only one of them. We ex- press $\Phi_{2}$, in terms of symmetric and antisymmetric tensors

$c^{\alpha}=\frac{\boldsymbol{b}^{\alpha}+\boldsymbol{b}^{\alpha T}}{2}, \quad \boldsymbol{d}^{\alpha}=\frac{\boldsymbol{b}^{\alpha}-\boldsymbol{b}^{\alpha T}}{2}$.

Then, we have

$\boldsymbol{b}^{\alpha}=c^{\alpha}+\boldsymbol{d}^{\alpha}, \quad \boldsymbol{b}^{\alpha T}=\boldsymbol{c}^{\alpha}-\boldsymbol{d}^{\alpha}$.

With this, $2 \Phi_{2}$ may be expressed in terms of the principal values $c^{\alpha}$, i.e.

$\Phi_{2}=\Phi_{21}+\Phi_{22}$

where,

$2 \Phi_{21}=A_{i j} c_{j}$,

$\Phi_{22}=\left(\gamma_{1}-\beta_{1}\right)\left(d_{1}^{2}+d_{2}^{2}+d_{3}^{2}\right)+\left(\gamma_{2}-\beta_{2}\right)\left(d_{4}^{2}+d_{5}^{2}+d_{6}^{2}\right)+4 \tau\left(d_{1} d_{4}+d_{2} d_{5}+d_{3} d_{6}\right)$,

$A_{1}=A_{11}=A_{22}=A_{33}=\alpha_{1}+\beta_{1}+\gamma_{1}, \quad A_{2}=A_{44}+A_{55}+A_{66}=\alpha_{2}+\beta_{2}+\gamma_{2}$,

$A_{12}=A_{23}=A_{31}=\alpha_{1}, \quad A_{14}=A_{25}=A_{36}=\tau, \quad A_{45}=A_{36}=A_{64}=\alpha_{2}$,

$c_{11}^{1}=c_{1}, \quad c_{22}^{1}=c_{2}, \quad c_{33}^{1}=c_{3}, \quad c_{11}^{2}=c_{4}, \quad c_{22}^{2}=c_{5}, \quad c_{33}^{2}=c_{6}$

$d_{12}^{1}=d_{1}, \quad d_{23}^{1}=d_{2}, \quad d_{31}^{1}=d_{3}, \quad d_{12}^{2}=d_{4}, \quad d_{23}^{2}=d_{5}, \quad d_{31}^{2}=d_{6}$.

From $\Phi_{22} \geq 0$, expressed by Eq. (61), it follows that

$\gamma_{1}-\beta_{1} \geq 0, \quad\left(\gamma_{1}-\beta_{1}\right)\left(\gamma_{2}-\beta_{2}\right)-4 \tau^{2} \geq 0$

$\Phi_{21}$ will be nonnegative, when the eigenvalues are nonnegative. Alternatively, all sub-determinants based on the diagonals of the following matrix are nonnegative:

$\begin{array}{cccccc}A_{1} & \alpha_{1} & \alpha_{1} & \tau & 0 & 0 \\ \alpha_{1} & A_{1} & \alpha_{1} & 0 & \tau & 0 \\ \alpha_{1} & \alpha_{1} & A_{1} & 0 & 0 & \tau \\ \tau & 0 & 0 & A_{2} & \alpha_{2} & \alpha_{2} \\ 0 & \tau & 0 & \alpha_{2} & A_{2} & \alpha_{2} \\ 0 & 0 & \tau & \alpha_{2} & \alpha_{2} & A_{2}\end{array}$

Hence, we obtain

$$
\begin{gathered}
\alpha_{1}+\beta_{1}+\gamma_{1} \geq 0, \quad \beta_{1}+\gamma_{1} \geq 0, \quad 2 \alpha_{1}+\beta_{1}+\gamma_{1} \geq 0 \\
\left(3 \alpha_{1}+\beta_{1}+\gamma_{1}\right)\left(3 \alpha_{2}+\beta_{2}+\gamma_{2}\right)-\tau^{2} \geq 0 \\
\left(\beta_{1}+\gamma_{1}\right)\left(3 \alpha_{1}+\beta_{1}+\gamma_{1}\right)\left(\alpha_{2}+\beta_{2}+\gamma_{2}\right) \\
-\left(2 \alpha_{1}+\beta_{1}+\gamma_{1}\right) \tau^{2} \geq 0
\end{gathered}
$$

$$
\begin{aligned}
\left(\beta_{1}+\gamma_{1}\right)^{2} & \left(3 \alpha_{1}+\beta_{1}+\gamma_{1}\right)\left(\beta_{2}+\gamma_{2}\right)\left(2 \alpha_{2}+\beta_{2}+\gamma_{2}\right) \\
& -2\left(\beta_{1}+\gamma_{1}\right)\left[\left(\beta_{1}+\gamma_{1}\right)\left(\alpha_{2}+\beta_{2}+\gamma_{2}\right)\right. \\
& \left.+\alpha_{1}\left(3 \alpha_{2}+2 \beta_{2}+2 \gamma_{2}\right)\right] \tau^{2} \\
& +\left(\alpha_{1}+\beta_{1}+\gamma_{1}\right) \tau^{4} \geq 0 .
\end{aligned}
$$

In the case of $\Phi_{1} \geq 0$, in Eq. (65), we replace $\alpha_{\alpha}$ by $\lambda_{\alpha}$, $\beta_{\alpha}$ by $\mu_{\alpha}, \gamma_{\alpha}$ by $\mu_{\alpha}+\kappa_{\alpha}$, and $\tau$ by $\sigma$.

With the inequalities Eqs. (56), (57), (63), (65), and these replacements, we will have $\Phi \geq 0$.

For simplicity and practical reasons, often twodimensional problems are treated. In this case, Eqs. (56), (57) and (63) are not changed. But the matrix (64) is reduced to

$$
\begin{array}{cccc}
A_{1} & \alpha_{1} & \tau & 0 \\
\alpha_{1} & A_{1} & 0 & \tau \\
\tau & 0 & A_{2} & \alpha_{2} \\
0 & \tau & \alpha_{2} & A_{2}
\end{array}
$$


In this case, we obtain the inequalities

$\alpha_{1}+\beta_{1}+\gamma_{1} \geq 0, \quad \beta_{1}+\gamma_{1} \geq 0$,

$2 \alpha_{1}+\beta_{1}+\gamma_{1} \geq 0, \quad\left(\beta_{1}+\gamma_{1}\right)\left(\beta_{2}+\gamma_{2}\right)-\tau^{2} \geq 0$,

$\left.2 \alpha_{1}+\beta_{1}+\gamma_{1}\right)\left(2 \alpha_{2}+\beta_{2}+\gamma_{2}\right)-\tau^{2} \geq 0$.

moduli are constants, we obtain the field equations

$$
\frac{\partial \rho^{1}}{\partial t}+\nabla \cdot\left(\rho^{1} \boldsymbol{v}^{1}\right)=0
$$

\section{Field equations}

Substituting the constitutive Eqs. (41), (43) and (55) into balance laws (17), (19) and (32), and assuming that all material

$$
\frac{\partial \rho^{2}}{\partial t}+\nabla \cdot\left(\rho^{2} \boldsymbol{v}^{2}\right)=0
$$

For small variations of temperature from a constant ambient temperature $T_{0}$, we can substitute

$\theta=T_{0}+T, \quad|T| \ll T_{0}$,

so that, we may replace $\nabla \theta / \theta$ by $\nabla T / T_{0}$, and take

$$
-\rho \theta \frac{\partial^{2} \psi}{\partial \theta^{2}} \simeq-\rho_{0} T_{0}\left(\frac{\partial^{2} \psi}{\partial T^{2}}\right)_{0}=c_{v},
$$

$(\rho)^{2} \theta \frac{\partial^{2} \psi}{\partial \theta \partial \rho} \simeq \rho_{0} T_{0}\left(\frac{\partial^{2} \psi}{\partial T \partial \rho}\right)_{0}=\delta$.

In the case of atmospheric fluid dynamics, we need to take into account the Coriolis acceleration. This is accomplished by replacing $\dot{\boldsymbol{v}}^{1}, \dot{\boldsymbol{v}}^{2}, \dot{\boldsymbol{v}}^{1}$ and $\dot{\boldsymbol{v}}^{2}$ as follows

$$
\begin{aligned}
& \dot{\boldsymbol{v}}^{1} \rightarrow \dot{\boldsymbol{v}}^{1}-2 \boldsymbol{\Omega} \times \boldsymbol{v}^{1}, \\
& \dot{\boldsymbol{v}}^{2} \rightarrow \dot{\boldsymbol{v}}^{2}-2 \boldsymbol{\Omega} \times \boldsymbol{v}^{2}, \\
& \dot{\boldsymbol{v}}^{1} \rightarrow \dot{\boldsymbol{v}}^{1}-\boldsymbol{\Omega} \times \boldsymbol{v}^{1}, \\
& \dot{\boldsymbol{v}}^{2} \rightarrow \dot{\boldsymbol{v}}^{2}-\boldsymbol{\Omega} \times \boldsymbol{v}^{2},
\end{aligned}
$$

where, $\boldsymbol{\Omega}$ is the rotation vector of Earth.

Field Eqs. (68) to (74) are expressed in rectangular coordinates. We expressed them in terms of vector operators, which are valid in orthogonal curvilinear coordinates.

$\frac{\partial \rho^{1}}{\partial t}+\nabla \cdot\left(\rho^{1} \boldsymbol{v}^{1}\right)=0$,

$\frac{\partial \rho^{2}}{\partial t}+\nabla \cdot\left(\rho^{2} \boldsymbol{v}^{2}\right)=0$, 


$$
\begin{aligned}
& -\nabla \pi^{1}+\left(\lambda_{1}+2 \mu_{1}+\kappa_{1}\right) \nabla \nabla \cdot \boldsymbol{v}^{1}-\left(\mu_{1}+\kappa_{1}\right) \nabla \times \nabla \times \boldsymbol{v}^{1}+\kappa_{1} \boldsymbol{\nabla} \times \boldsymbol{v}^{1}+\sigma\left(\nabla \nabla \cdot \boldsymbol{v}^{2}\right. \\
& \left.-\nabla \times \nabla \times v^{2}+\nabla \times v^{2}\right)-\xi\left(v^{1}-v^{2}\right)-\frac{\zeta}{T_{0}} \nabla T+\rho 1\left(f^{1}-\dot{\boldsymbol{v}}^{1}+2 \boldsymbol{\Omega} \times \boldsymbol{v}^{1}\right)=\mathbf{0}, \\
& -\nabla \pi^{2}+\left(\lambda_{2}+2 \mu_{2}+\kappa_{2}\right) \nabla \nabla \cdot v^{2}-\left(\mu_{2}+\kappa_{2}\right) \nabla \times \nabla \times v^{2}+\kappa_{2} \nabla \times v^{2}+\sigma\left(\nabla \nabla \cdot v^{1}\right. \\
& \left.-\nabla \times \nabla \times v^{1}+\nabla \times v^{1}\right)+\xi\left(v^{1}-v^{2}\right)+\frac{\zeta}{T_{0}} \nabla T+\rho^{2}\left(f^{2}-\dot{\boldsymbol{v}}^{2}+2 \boldsymbol{\Omega} \times \boldsymbol{v}^{2}\right)=\mathbf{0}, \\
& \left(\alpha_{1}+\beta_{1}+\gamma_{1}\right) \nabla \nabla \cdot v^{1}-\gamma_{1} \nabla \times \nabla \times v^{1}+\tau \nabla \nabla \cdot v^{2}+\kappa_{1}\left(\nabla \times v^{1}-2 v^{1}\right) \\
& +\sigma\left(\nabla \times \boldsymbol{v}^{2}-2 \boldsymbol{v}^{2}\right)-\varpi\left(\boldsymbol{v}^{1}-\boldsymbol{v}^{2}\right)+\rho^{1}\left(\boldsymbol{l}^{1}-j^{1} \dot{\boldsymbol{v}}^{1}+j^{1} \boldsymbol{\Omega} \times \boldsymbol{v}^{1}\right)=\mathbf{0}, \\
& \left(\alpha_{2}+\beta_{2}+\gamma_{2}\right) \nabla \nabla \cdot v^{2}-\gamma_{2} \nabla \times \nabla \times v^{2}+\tau \nabla \nabla \cdot v^{1}+\kappa_{2}\left(\nabla \times v^{2}-2 v^{2}\right) \\
& +\sigma\left(\boldsymbol{\nabla} \times \boldsymbol{v}^{1}-2 \boldsymbol{v}^{1}\right)+\varpi\left(\boldsymbol{v}^{1}-\boldsymbol{v}^{2}\right)+\rho^{2}\left(\boldsymbol{l}^{2}-j^{2} \dot{\boldsymbol{v}}^{2}+j^{2} \boldsymbol{\Omega} \times \boldsymbol{v}^{2}\right)=\mathbf{0}, \\
& -c_{v} \dot{T}-\delta \nabla \cdot\left(\boldsymbol{v}^{1}+\boldsymbol{v}^{2}\right)+\sum_{\alpha}\left({ }_{D} \boldsymbol{t}^{\alpha} \cdot \boldsymbol{a}^{\alpha T}+\boldsymbol{m}^{\alpha} \cdot \boldsymbol{b}^{\alpha}\right)+\left[\xi\left(\boldsymbol{v}^{1}-\boldsymbol{v}^{2}\right)+\varsigma \frac{\nabla T}{T_{0}}\right] \cdot\left(\boldsymbol{v}^{1}-\boldsymbol{v}^{2}\right) \\
& +\varpi\left(v^{1}-v^{2}\right) \cdot\left(v^{1}-v^{2}\right)+\nabla \cdot\left[\varsigma\left(v^{1}-v^{2}\right)+\frac{K}{T_{0}} \nabla T\right]+\rho h=0 .
\end{aligned}
$$

In Eq. (85), ${ }_{D} \boldsymbol{t}^{\alpha}, \boldsymbol{a}^{\alpha}, \boldsymbol{m}^{\alpha}, \boldsymbol{b}^{\alpha}, \boldsymbol{v}^{\alpha}$ and $\boldsymbol{v}^{\alpha}$ denote the physical components of tensors.

Field Eqs. (79) to (85) constitute a system of fifteen partial differential equations to determine the fifteen unknown functions $\rho_{1}, \rho_{2}, \boldsymbol{v}^{1}, \boldsymbol{v}^{2}, \boldsymbol{v}^{1}, \boldsymbol{v}^{2}$ and $T$, given the external loads $\boldsymbol{f}^{1}, \boldsymbol{f}^{2}, \boldsymbol{l}^{1}, \boldsymbol{l}^{2}$ and $h$. Note that partial pressures $\pi^{1}, \pi^{2}$ and $c_{v}$ and $\delta$, given by Eqs. (43), (76) and (77), are determined when the the free energy function $\psi$ is specified. Thus, the system (79) to (85) is closed. Under appropriate boundary and initial conditions, the field equations may be solved to determine the unknown functions.

\section{Boundary Conditions}

Let $\bar{V}$ denote a regular region of the Euclidean space, occupied by the mixture, whose boundary is $\partial V$. The interior of $\bar{V}$ is denoted by $V$, and the exterior unit normal to $\partial V$ is denoted by $\boldsymbol{n}$. Let $S_{i}(i=1,2 \ldots, 6)$ denote subsets of $\partial V$, such that

$$
\bar{S}_{1} \cup S_{2}=\bar{S}_{3} \cup S_{4}=\bar{S}_{5} \cup S_{6}=\partial V,
$$$$
S_{1} \cap S_{2}=S_{3} \cap S_{4}=S_{5} \cap S_{6}=0 \text {. }
$$

A mixed set of boundary conditions on the surfaces, at time $T^{+}=[0, \infty)$, may be expressed as:

$$
\begin{array}{rlll}
\boldsymbol{v}^{\alpha}=\boldsymbol{v}_{0}^{\alpha} & \text { on } & \bar{S}_{1} \times T^{+}, & t_{k l}^{\alpha} n_{k}^{\alpha}=t_{0 l}^{\alpha} \quad \text { on } S_{2} \times T^{+}, \\
\boldsymbol{v}^{\alpha}=\boldsymbol{v}_{0}^{\alpha} & \text { on } & \bar{S}_{3} \times T^{+}, & m_{k l}^{\alpha} n_{k}^{\alpha}=m_{0 l}^{\alpha} \text { on } S_{4} \times T^{+}, \\
T=T_{0} & \text { on } & \bar{S}_{5} \times T^{+}, & q_{k} n_{k}^{\alpha}=q_{0}^{\alpha} \quad \text { on } S_{6} \times T^{+},
\end{array}
$$

where, $\boldsymbol{v}_{0}^{\alpha}, \boldsymbol{v}_{0}^{\alpha}, t_{0 l}^{\alpha}, m_{0 l}^{\alpha}, T_{0}$ and $q_{0}^{\alpha}$ are prescribed. When any one of the surfaces is absent, the accompanying boundary conditions are extended to cover $\partial V$. For example, when $S_{2}=0, \bar{S}_{1}$, becomes $\partial V$, and when $S_{4}=0, \bar{S}_{3}$ becomes $\partial V$, so that

$\boldsymbol{v}^{\alpha}=\boldsymbol{v}_{0}^{\alpha}, \quad \boldsymbol{v}^{\alpha}=\boldsymbol{v}_{0}^{\alpha} \quad$ on $\quad \partial V$.
These conditions imply strict adherence on the boundary. Initial Conditions

The initial conditions usually consist of Cauchy data, expressed by

$\rho(x, 0)=\rho^{0}(\boldsymbol{x}), \quad \boldsymbol{v}^{\alpha}(\boldsymbol{x}, 0)=\boldsymbol{v}^{\alpha 0}(\boldsymbol{x})$,

$\boldsymbol{v}^{\alpha}(\boldsymbol{x}, 0)=v^{\alpha 0}(\boldsymbol{x}), \quad T(\boldsymbol{x}, 0)=T^{0}(\boldsymbol{x})$ in $V$,

where, quantities carrying a superscript $\left(^{0}\right)$ are prescribed throughout the body, at time $t=0$.

\section{Incompressible fluids}

In incompressible fluids, $\rho^{\alpha}=\rho_{0}^{\alpha}=$ const . and we have

$\boldsymbol{\nabla} \cdot \boldsymbol{v}^{\alpha}=0, \quad \boldsymbol{\nabla} \cdot \boldsymbol{v}^{\alpha}=0$.

The pressures $\pi^{\alpha}$ are now replaced by an unknown pressure $p^{\alpha}(\boldsymbol{x}, t)$ and the field equations are simplified by using (5.23).

\section{Mixtures of two Newtonian fluids}

When the constituent fluids can be approximated by Newtonian fluids, basic equations are greatly simplified. In this case, the field equations follow, by setting $\kappa_{1}=\kappa_{2}=v^{1}=v^{2}=$ 0 , and ignoring the remainder of the Eqs. (83) and (84), since these equations arise from the nonsymmetry of the stress tensor and the existence of the couple stress.

$\frac{\partial \rho^{1}}{\partial t}+\nabla \cdot\left(\rho^{1} \boldsymbol{v}^{1}\right)=0$,

$\frac{\partial \rho^{2}}{\partial t}+\nabla \cdot\left(\rho^{2} \boldsymbol{v}^{2}\right)=0$, 


$$
\begin{array}{r}
-\nabla \pi^{1}+\left(\lambda_{1}+2 \mu_{1}\right) \nabla \nabla \cdot v^{1}-\mu_{1} \nabla \times \nabla \times v^{1}+\sigma\left(\nabla \nabla \cdot v^{2}-\nabla \times \nabla \times v^{2}\right) \\
-\xi\left(\boldsymbol{v}^{1}-\boldsymbol{v}^{2}\right)-\frac{\varsigma}{T_{0}} \nabla T+\rho^{1}\left(\boldsymbol{f}^{1}-\dot{\boldsymbol{v}}^{1}+2 \boldsymbol{\Omega} \times \boldsymbol{v}^{1}\right)=\mathbf{0}, \\
-\nabla \pi^{2}+\left(\lambda_{2}+2 \mu_{2}\right) \nabla \nabla \cdot \boldsymbol{v}^{2}-\mu_{2} \nabla \times \nabla \times \boldsymbol{v}^{2}+\sigma\left(\nabla \nabla \cdot \boldsymbol{v}^{1}-\nabla \times \nabla \times \boldsymbol{v}^{1}\right) \\
+\xi\left(\boldsymbol{v}^{1}-\boldsymbol{v}^{2}\right)+\frac{\varsigma}{T_{0}} \nabla T+\rho^{2}\left(\boldsymbol{f}^{2}-\dot{\boldsymbol{v}}^{2}+2 \boldsymbol{\Omega} \times \boldsymbol{v}^{2}\right)=\mathbf{0}, \\
-c_{v} \dot{T}-\delta \nabla \cdot\left(\boldsymbol{v}^{1}+\boldsymbol{v}^{2}\right)+{ }_{D} \boldsymbol{t}^{1} \cdot \boldsymbol{d}^{1 T}+{ }_{D} \boldsymbol{t}^{2} \cdot \boldsymbol{d}^{2 T}+\left[\xi\left(\boldsymbol{v}^{1}-\boldsymbol{v}^{2}\right)+\varsigma \frac{\nabla T}{T_{0}}\right] \cdot\left(\boldsymbol{v}^{1}-\boldsymbol{v}^{2}\right) \\
+\nabla \cdot\left[\zeta\left(\boldsymbol{v}^{1}-\boldsymbol{v}^{2}\right)+\frac{K}{T_{0}} \nabla T\right]+\rho h=0,
\end{array}
$$

where,

$\boldsymbol{t}^{\alpha}=-\pi^{\alpha} \mathbf{1}+\lambda_{\alpha} \boldsymbol{\nabla} \cdot \boldsymbol{v}^{\alpha}+2 \mu_{\alpha} \boldsymbol{d}^{\alpha}$,

$d_{k l}^{\alpha}=\frac{1}{2}\left(v_{k, l}^{\alpha}+v_{l, k}^{\alpha}\right)$

\section{Boundary Conditions}

The boundary conditions follow from Eq. (87), by dropping conditions on $\boldsymbol{v}^{\alpha}$, and $m_{k l}^{\alpha}$.

$$
\begin{aligned}
& \boldsymbol{v}^{\alpha}=\boldsymbol{v}_{0}^{\alpha} \quad \text { on } \quad \bar{S}_{1} \times T^{+}, t_{k l}^{\alpha} n_{k}^{\alpha}=t_{0 l}^{\alpha} \quad \text { on } \quad S_{2} \times T^{+} \text {, } \\
& T=T_{0} \quad \text { on } \quad \bar{S}_{3} \times T^{+}, q_{k} n_{k}^{\alpha}=q_{0}^{\alpha} \quad \text { on } \quad S_{4} \times T^{+} \text {, }
\end{aligned}
$$

where, $\bar{S}_{1} \cup S_{2}=\bar{S}_{3} \cup S_{4}=\partial V, S_{1} \cap S_{2}=S_{3} \cap S_{4}=0$.

\section{Initial Conditions}

$$
\begin{aligned}
& \rho(\boldsymbol{x}, 0)=\rho^{0}(\boldsymbol{x}), \quad \boldsymbol{v}^{\alpha}(\boldsymbol{x}, 0)=\boldsymbol{v}^{\alpha 0}(\boldsymbol{x}), \\
& T(\boldsymbol{x}, 0)=T^{0}(\boldsymbol{x}) \quad \text { in } \quad V .
\end{aligned}
$$

\section{Conclusion}

We have introduced a continuum theory of atmospheric fluids. Any binary combinations of rain, snow, sand and ice are considered a mixture of two micropolar fluids at one average temperature. The balance laws are given, and thermodynamically admissible constitutive equations are constructed for a mixture of two micropolar fluids. Thermodynamic restrictions on the material moduli are investigated. Field equations are obtained and boundary and initial conditions are stated. As a special case of the micropolar mixture, we obtained the field equations of a mixture of two Newtonian fluids.

The field equations can be used to predict atmospheric phenomena. These include the predictions of densities, velocities, intrinsic rotations, diffusions of species and the mean temperature of atmospheric mixtures. The theory is applicable to oceanic boundary-initial value problems as well.
From the field Eqs. (81) to (85), it is clear that strong couplings exist among the velocities and gyrations of species. Consequently, the gyrations will alter the velocities and vice versa. The constituent fluids display couple stress and a nonsymmetric stress tensor.

As compared to the binary mixtures of Newtonian fluids (Eqs. 93 to 95), here, we encounter an additional physical phenomena, diffusion due to the difference of gyrations. This effect also guides the conduction of heat in the mixture.

Edited by: S. Wiggins

Reviewed by: two referees

\section{References}

Bowen, R. M.: Theory of Mixtures, Continuum Physics III, edited by Eringen, A. C., Academic Press, New York, 1-127, 1976.

Edelen, D. G. B.: College Lectures on Thermodynamics, Texas A and M University, Austin, Texas, 1993.

Eringen, A. C.: Mechanics of Continua, (Second Edition), Robert E. Krieger Publishing Co., Melbourn, Florida, 151, 1980.

Eringen, A. C.: A Continuum Theory of Swelling Porous Elastic Soils, Int. J. Engng. Sci., 32, 1337-1349, 1994.

Eringen, A. C.: Microcontinuum Field Theories, II, Fluent Media, Springer-Verlag, New York, 8, 2001.

Eringen, A. C. and Ingram, J. D.: A continuum Theory of Chemically Reacting Media-I, Int. J. EngIng. Sci., 3, 197-212, 1965.

Kirwan, A. D. Jr. and Chang, M. S.: On the Micropolar Ekman Problem, Int. J. Engng. Sci., 14, 685-692, 1976.

Twiss, R. J. and Eringen, A. C.: Theory of Mixture for Micromorphic Materials, I. Balance Laws, Int. J. Engng. Sci., 9, 10191044, 1971.

Twiss, R. J. and Eringen, A. C.: Theory of Mixture for Micromorphic Materials, II. Elastic Constitutive Equations, Int. J.Engng. Sci., 10, 437-465, 1972.

Wilmanski, K.: Some Questions on Material Objectivity Arising in Models of Porous Materials, Rational Continua, Classical and New, edited by Podio-Guidugli, P. and Brocato, M., SpringerVerlag, Italia, 183-195, 2003. 